\title{
Prevalence \& features of inner ear malformations among children with congenital sensorineural hearing loss: A Public Cochlear Implant Centre Experience
}

\author{
Jawwad Ahmed ${ }^{1}$, Ghulam Saqulain ${ }^{2}$, \\ Muhammad lqbal Javed Khan ${ }^{3}$, Mobeen Kausar ${ }^{4}$
}

\begin{abstract}
Objective: To determine the prevalence and features of inner ear anomalies in children with congenital profound hearing loss who presented at our cochlear implant center based on imaging studies.

Methods: This retrospective study reviewed charts of children with congenital SNHL, who presented to Department of Otolaryngology \& Auditory Implant Centre, Capital Hospital Islamabad over a period of 2 years from $1^{\text {st }}$ May 2017 to $30^{\text {th }}$ April 2019. These included 481 cases of both genders aged between 1 to 12 years. After gathering demographic data, audiological data, computed tomography findings of the temporal bone were analyzed. Data was analyzed using SPSS 22.

Results: The Inner Ear Malformations were identified in 48(10\%) children including $28(58.33 \%)$ males and $20(41.67 \%)$ female. Most $20(41.67 \%)$ presented at $>3-5$ years of age followed by $19(39.58 \%)$ at $2-3$ years. However, no significant association of gender $(p=0.57, p=0.076)$ and age of presentation $(p=0.344, p=0.697)$ for right and left ears was noted with inner ear malformations. The most common anomaly noted were $\mathrm{CLA}, \mathrm{CH}-\mathrm{III}$ and $\mathrm{CH}-\mathrm{Il}$ in decreasing order of frequency in both ears.

Conclusion: The prevalence of IEM's was found to be 48(10\%). Commonest anomalies noted were CLA, CH$\mathrm{III}$ and $\mathrm{CH}-\mathrm{II}$. No significant association of gender and age of presentation was noted with type of anomaly in both ears.
\end{abstract}

KEYWORDS: Computed tomography scan, Middle ear malformations, Prevalence.

doi: https://doi.org/10.12669/pjms.36.7.3134

How to cite this:

Ahmed J, Saqulain G, Khan MIJ, Kausar M. Prevalence \& features of inner ear malformations among children with congenital sensorineural hearing loss: A Public Cochlear Implant Centre Experience . Pak J Med Sci. 2020;36(7):1511-1516. doi: https://doi.org/10.12669/pjms.36.7.3134

This is an Open Access article distributed under the terms of the Creative Commons Attribution License (http://creativecommons.org/licenses/by/3.0), which permits unrestricted use, distribution, and reproduction in any medium, provided the original work is properly cited.

\section{INTRODUCTION}

Cochlear implant services for the deaf children got established in Pakistan over the last two decades, however no local studies report the prevalence of inner ear malformations. This is

Correspondence:

Dr. Ghulam Saqulain, FCPS.

Head of Department,

Department of Otolaryngology,

Capital Hospital PGMI,

G 6/2, Islamabad, Pakistan.

Email: ghulam_saqulain@yahoo.com

* Received for Publication:

* Accepted for Publication:
June 26, 2020

August 23, 2020 important in planning surgical treatment and predicting prognosis.

With a local prevalence of congenital anomalies of $7 \%$, around $11.94 \%$ suffer from malformations of face, neck, eyes and ears. ${ }^{1}$ Inner ear anomalies are usually encountered while diagnosing and operating pediatric cases with non-serviceable hearing and both membranous and bony malformations may be encountered. ${ }^{2}$ Joshi VMetal, has reported a high prevalence of IEMs (15 to 20\%) in cases with severe or profound hearing loss $(\mathrm{HL})^{3}$ with fear of intra-operative complications. Hence identification of bony anomalies is important in planning cochlear implants (CI) surgery. ${ }^{4}$ In spite of the fact that hearing and speech development 
do not differ in post implant cases with and without IEMs ${ }^{5}$, however more difficulties may be faced with IEM's including gusher and facial nerve abnormalities. ${ }^{6}$

Evaluation of cochlear implant candidates requires imaging studies in addition to audiological investigations to delineate anomalies of the temporal bone and membranous structures like $8^{\text {th }}$ cranial nerve $(\mathrm{CN})$, inner ear (IE), middle ear (ME) and outer ear (OE). High-resolution computed tomography (CT) scan and Magnetic resonance imaging (MRI) studies are gold standard imaging studies required in such cases and used in conjunction with each other. ${ }^{7}$ Among other structures, a High-resolution CT can help visualize coexisting ME and OE malformations and anatomic variants, while MRI has more sensitivity for delineating $8^{\text {th }} \mathrm{CN}$ and spaces in the IE filled with perilymph and endolymph and brain anomalies. ${ }^{3}$

A variety of congenital anomalies are seen both syndromic and non-syndromic ${ }^{7}$, with varying presentation, radiological and audiological features and present with different findings on surgical intervention. ${ }^{2}$ These anomalies have been grouped into eight groups by Bajin $\mathrm{SL}^{2}$, including Complete aplasia of labyrinth; Rudimentary otocyst; Cochlear aplasia; Common cavity; Cochlear hypoplasia; Incomplete partition-I, Incomplete partition-II, Incomplete partitionIII; Enlarged vestibular aqueduct; and Cochlear aperture abnormalities. The bony labyrinth originates from mesoderm and therefore its malformations are noted as deficient coils and branching of tubular structures derived from otocyst. Also there may be aplasia or dysplasia of sensory epithelium, and deficient cartilaginous and bony framework.

Frequency of congenital HL in Pakistan has been reported by Ahmed S et al. as 13 per 1000 with $15 \%$ profound and $85 \%$ moderate to severe $\mathrm{HL}$, which is quite high compared to other parts of the world $^{8}$, with the first cochlear implantation in a case of IEM in Pakistan reported in 2013. ${ }^{9}$

The richness of HL gene heterogeneity in Pakistani population makes it quite attractive for research ${ }^{10}$, combined with higher prevalence of congenital HL, and establishing of cochlear implant facility in the public sector, prompted and facilitated this study to determine the prevalence and features of inner ear anomalies in children with congenital profound hearing loss who presented at out cochlear implant center based on imaging studies. With this subject lacking in local literature in Pakistan, this study is of immense importance keeping in view high prevalence of HL in Pakistan. Also this could be helpful in providing reliable statistical data regarding IEM's in patients with congenital HL and will be helpful to plan effective treatment strategies and for research purposes.

\section{METHODS}

This retrospective study reviewed medical charts of children with Sensorineural hearing loss (SNHL), who presented to or were referred to Department of Otolaryngology \& Cochlear implant Centre, Capital Hospital, Islamabad, over a period of 2 years from 1st May 2017 to 30th April 2019. These included $\mathrm{N}=481$ cases of both genders aged between one to 12 years. After gathering demographic data, audiological data, computed tomography findings of the temporal bone were analyzed. Study was conducted after obtaining ethical approval of the research from Ethical Review Committee of Capital Hospital PGMI, Islamabad vide Ref. No. 2029-05-002 dated 7th May, 2020.

After collection, data was tabulation using Microsoft Excel Worksheet and analyzed statistically using Statistical Package for Social Studies (SPSS) Version-23. Results were analyzed using descriptive statistic including frequencies, percentages, mean, and standard deviation. Chi-square test was used to see gender and age association. The data was then viewed along with national and international literature and resulting deductions were discussed.

\section{RESULTS}

Current study included 286(59.5\%) males and $195(40.5 \%)$ females with a mean age of $3.62 \pm 2.29$ years. The frequency of middle ear anomalies was $48(10 \%)$. Of the 48 Right Ears, 5(10.4\%) were normal, the commonest anomaly was CLA 9(18.8\%), followed by $\mathrm{CH}-\mathrm{III} 8(16.7 \%)$ and $\mathrm{CH}-\mathrm{II}$ $5(10.4 \%)$ (Table-I). Of the 48 Left Ears, 7(14.6\%) were normal and commonest anomaly noted was CLA \& CH-III 8(16.7\%) each, followed by CH-II in $6(12.5 \%)$ cases. Individual anatomic deformities noted are summarized in Table-II.

As regards the different IEM's noted in in the current study, out of the 48 Right Ears, the commonest anomaly being CLA 9(18.8\%), followed by CH-III $8(16.7 \%)$ and $\mathrm{CH}-\mathrm{II} 5(10.4 \%)$. While on the left side, $7(14.6 \%)$ were normal ears 
Inner ear malformations in children with congenital sensorineural hearing loss

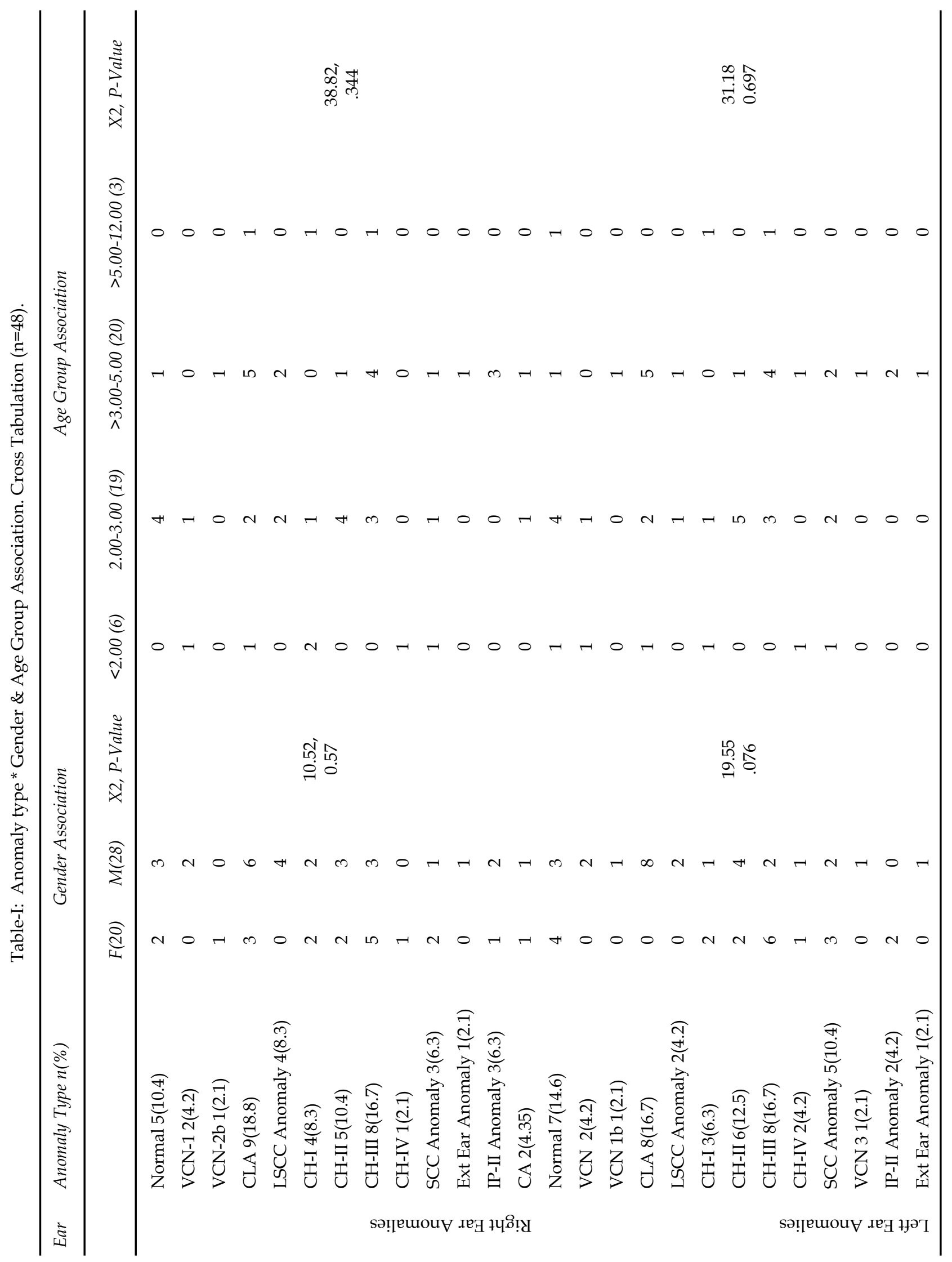

$\begin{array}{lllll}\text { Pak J Med Sci } \quad \text { November - December } 2020 & \text { Vol. } 36 & \text { No. } 7 & \text { www.pjms.org.pk } 1513\end{array}$ 
Table-II: Individual congenital anatomic deformities * Ear. Cross tabulation. $(\mathrm{N}=48)$.

\begin{tabular}{llcc}
\hline Congenital Anatomic Deformity & \multicolumn{2}{c}{ Ear } \\
\hline Area & Anomaly & Right n (\%) & Left n (\%) \\
\hline VIII N & Normal & $27(56.3)$ & $29(60.4)$ \\
& Absent & $16(33.3)$ & $14(29.2)$ \\
& Thin & $5(10.4)$ & $5(10.4)$ \\
IAC & Normal & $25(52.1)$ & $28(58.3)$ \\
& Reduced & $13(27.1)$ & $9(18.8)$ \\
& Absent & $10(20.8)$ & $11(22.9)$ \\
LSCC & Normal & $12(25)$ & $17(35.4)$ \\
& Absent & $10(20.8)$ & $12(25)$ \\
& Small Hypoplastic & $26(54.2)$ & $19(39.6)$ \\
PSCC & Normal & $23(47.9)$. & $25(52.1)$ \\
& Absent & $17(37.4)$ & $15(31.3)$ \\
& Small Hypoplastic & $8(16.7)$ & $8(16.7)$ \\
SSCC & Normal & $25(52.1)$ & $28(58.3)$ \\
& Absent & $18(37.5)$ & $16(33.3)$ \\
& Small Hypoplastic & $5(10.4)$ & $4(8.3)$ \\
Cochlea & Normal & $16(33.3)$ & $19(39.6)$ \\
& Absent & $13(27.1)$ & $9(18.8)$ \\
& Sac & $12(25)$ & $12(25)$ \\
& Apical turn absent & $5(10.4)$ & $4(8.3)$ \\
& Only basal turn present & $2(4.2)$ & $4(8.3)$ \\
\hline
\end{tabular}

and the commonest anomaly noted was CLA \& CH-III $8(16.7 \%)$ each, followed by $\mathrm{CH}-\mathrm{II}$ in $6(12.5 \%)$ cases. As regards individual anatomic deformities noted, $8^{\text {th }}$ Nerve was absent in $16(33.3 \%)$ right ears and $14(29.2 \%)$ left ears while it was thin in $5(10.4 \%)$ of both right and left ears. Internal Auditory Canal (IAC) was reduced in size in $13(27.1 \%) \& 9(18.8 \%)$ and absent in $10(20.8 \%)$ \& $11(22.9 \%)$ of Right and Left ears respectively. Lateral Semi Circular Canal was absent in $10(20.8 \%)$ \& $12(25 \%)$; and small hypoplastic in 26(54.2\%) \& 19(39.6\%) of Right and Left ears respectively. PSCC was absent in 17(37.4\%) $\& 15(31.3 \%)$ of right and left ears; and small hypoplastic in $8(16.7 \%)$ of both ears each. SSCC was absent in $18(37.5 \%)$ \& $16(33.3 \%)$; and small hypoplastic in $4(10.4 \%)$ \& $4(8.3 \%)$ of Right and left ears each. Cochlea was absent in $13(27.1 \%) \& 9(18.8 \%)$; apical turn was absent in $5(10.4 \%) \& 498.3 \%)$ and only basal turn was present in $2(4.2 \%) \& 4(8.3 \%)$ of right and left ears each. While cochlea was just a Sac in $12(25 \%)$ of both ears.

There was no significant association of type of anomaly with gender in both right and left ear with $p=0.57 \& p=0.076$ respectively. Also no association of type of anomaly was noted for age with $\mathrm{p}=0.344$ $\& p=0.697$ in right and left ear respectively.

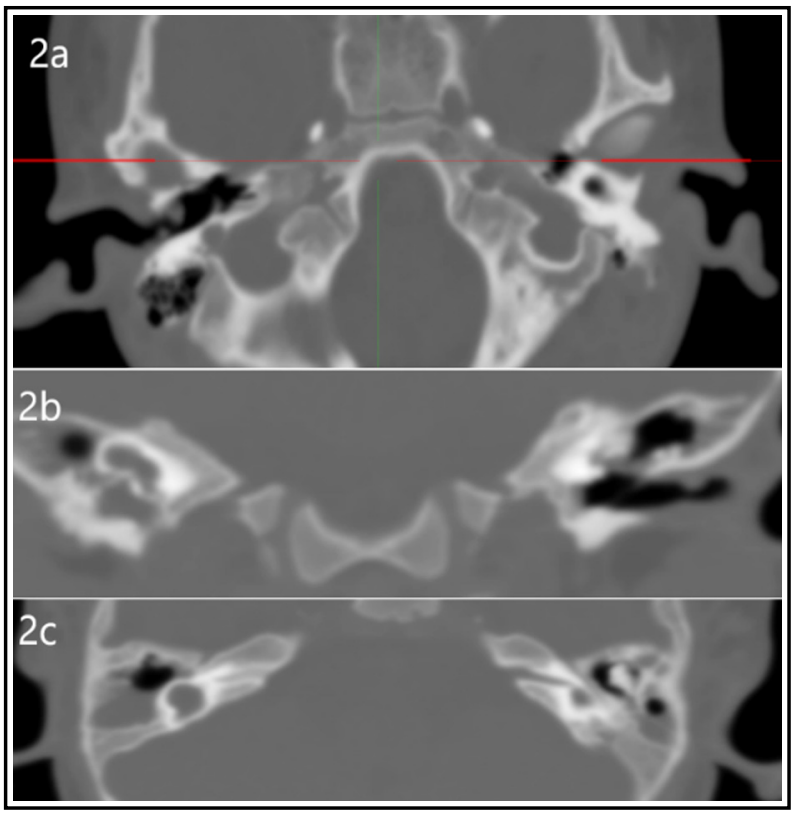

Fig.1: Computed Tomography Scan Axial view showing a) Congenital absence of inner ear \& Vth Cranial Nerve bilaterally consistent with CLA, b \& c) Hypoplasia of basal turns with $6 \mathrm{~mm}$ on right side while $4 \mathrm{~mm}$ on left side with a cyst like structure replacing Semicircular canals consistent with $\mathrm{CH} 3$.

\section{DISCUSSION}

Current study with 286(59.5\%) males and 195(40.5\%) females, did not reveal any significant association of type of anomaly with gender. In contrast, Zanon A et al. in their review noted that Gender difference with regards to balance, hearing, speech and IEM's exist, however studies regarding gender prevalence of different malformations are lacking ${ }^{11}$, with the exception that high prevalence of outer ear anomalies in males. ${ }^{12}$

With a mean age of $3.62 \pm 2.29$ years and an age range of 1 to 12 years, the majority of IEM $(n=20)$ were reported in age group $>3$ to 5 years, followed by $n=19$ in age group 2-3 years, however there was no significant age association with IEM. In contrast Masuda $S$ et al. reported that the prevalence of malformations was significantly $(\mathrm{p}<0.01)$ higher in infants $(84.6 \%)$ compared to those 1 to 15 years age $(55.8 \%) .^{13}$

We noted a high frequency of IEM's $48 / 481(10 \%)$ in cases with bilateral SNHL. While, a Saudi study by Aldhefeeri \& Alsanosi reported a slightly lower prevalence of $7.5 \% .{ }^{14}$ International literature shows a highly variable prevalence of IEM's. In sheer contrast to our study, Masuda and Usui in 2019 reported a high prevalence of $24.3 \%$ 
in cases with Bilateral SNHL, while they noted a low prevalence of $3.7 \%$ in cases Unilateral SNHL. ${ }^{15}$ Also a Chinese study by Sun B et al. ${ }^{16}$ reported an incidence of $30.69 \%$. In contrast, Agarwal SK et al. reported a frequency of $13.93 \%$, indicating that probably population in this subcontinent was less prone to develop IEM's. ${ }^{17}$

Large variations in the results of different studies have been reported. In a Saudi study by Aldhefeeri \& Alsanosi Large vestibular aqueduct was commonest pathology (33.3\%), followed by dysplasia of SCC (29.1\%) and cochlear hypoplasia $(4.1 \%) .{ }^{14}$ While in another study of Chinese origin, by Sun B et al. revealed malformations of cochlea $(31 \%)$, simple vestibular aqueduct $(40.33 \%)$, and simple vestibular aqueduct/SCC/IAC in $7.35 \%$. Of the malformations of the cochlea Michel deformity was noted in $1.13 \%$, cochlea aplasia in $1.81 \%$, common cavity deformity in $3.17 \%$, incomplete partition type I in $8.62 \%$, cochlea hypoplasia in $9.07 \%$ and incomplete partition type II was reported to be present in $76.19 \%{ }^{16}$ While in an Indian study by Agarwal SK et al. the prevalence of cochlear anomaly was $73 \%$, $87.1 \%$ had vestibular malformations, $56.4 \%$ had vestibular aqueduct malformation, $30.7 \%$ were with IAC anomaly and $29.4 \%$ had $8^{\text {th }} \mathrm{CN}$ anomalies. ${ }^{17}$ In an imaging study by El Sheikh $\mathrm{E}$ et al. cochlear hypoplasia the commonest $(4.5 \%)$, followed by common cavity $(3 \%)$, IP-1 (3\%), IP-2 (16.7\%), IP-3 (7.6\%), posterior rotated cochlea $(6.1 \%)$, dilated vestibule $(3 \%)$, isolated SCC hypoplasia $(3.03 \%)$, SCC hypoplasia with common cavity $(3.03 \%)$, dysplastic SCC $(6.8 \%)$, dilated IAC (4.5\%), hypo-plastic IAC $(18.2 \%)$, VCN hypoplasia (18.2\%), isolated EVA $(36.4 \%) .{ }^{18}$ Also in a study by Dhanasingh A, found normal anatomy in four, enlarged vestibular aqueduct in 3 , cochlear aplasia in 2, IP-I in 8, IP-II in 3, IPIII in four, $\mathrm{CH}$ in 17, common cavity (CC) in 5. Majority of $\mathrm{CH}$ cases had cochlear height shorter than four $\mathrm{mm}$ whereas the $\mathrm{CC}$ cases measured cochlear height above $6 \mathrm{~mm}$. For all the other malformation types, cochlear height was between 4 and $6 \mathrm{~mm} \cdot{ }^{19}$

Also in another study, 32(46.4\%) had stenosis of $8^{\text {th }}$ nerve canal out of which 13 had canal stenosis alone. While IAC anomalies were seen in 22(31.8\%), cochlear anomalies in $14(20.3 \%)$ and vestibular and SCC anomalies in 5(7.2\%), enlarged vestibular aqueduct in $2(2.9 \%)$. The prevalence of narrowed internal auditory canal was significantly more in cases with $8^{\text {th }}$ nerve canal stenosis. ${ }^{13} Y \mathrm{Y} \mathrm{JS}$ et al. reported that $57 \%$ cases of unilateral SNHL had $8^{\text {th }}$ nerve canal atresia or stenosis associated with inner ear malformations..$^{20}$ Shama SAM et al. reported that aplasia commonly involves cochlea $(26.9 \%)$, and semicircular canals $(19.23 \%)$, while dysplasia commonly involves Vestibule. ${ }^{21}$

\section{CONCLUSIONS}

The prevalence of IEM's of $48 / 481(10 \%)$ in the current study was not very high. Commonest anomaly noted were CLA (fig.1a), CH-III (Fig.1b,c) and CH-II. No significant association of gender and age at presentation noted with type of anomaly in both ears.

\section{Conflict of Interest: None.}

Grant Support \& Financial Disclosures: None.

\section{REFERENCES}

1. Hussain S, Asghar I, Sabir MUD, chattha MN, Tarar $\mathrm{SH}$, Mushtaq R. Prevalence and pattern of congenital malformations among neonates in the neonatal unit of a teaching hospital. J Pak Med Assoc. 2014;64(6):629-634.

2. Sennaroglu L, Bajin MD. Classification and Current Management of Inner Ear Malformations. Balkan Med J. 2017;34(5):397-411.

3. Joshi VM, Navlekar SK, Kishore GR, Reddy KJ, Kumar ECV. CT MR. Imaging of the Inner Ear and Brain in Children with Congenital Sensorineural Hearing Los. Radio Graphics. 2012;32:683-698.

4. Quirk B, Youssef A, Ganau M, D'Arco F. Radiological diagnosis of the inner ear malformations in children with sensorineural hearing loss. Br J Open. 2019;1(1): doi: 10.1259/bjro.20180050

5. Celik M, Karatas E, Kanlikama M. Outcomes of cochlear implantation in children with and without inner ear malformations. Pak J Med Sci. 2018;34(2):380-384.

6. Farhood Z, Nguyen SA, Miller SC, Holcomb MA, Meyer TA, Rizk HG. Cochlear Implantation in Inner Ear Malformations: Systematic Review of Speech Perception Outcomes and Intraoperative Findings. Otolaryngol Head Neck Surg. 2017;156(5):783-793.

7. Sepahdari AR, Zipser BD, Pakdaman MN. Imaging of congenital temporal bone anomalies. Oper Tech Otolayngol Head Neck Surg. 2014;25(1):13-20.

8. Ahmed S, Sheraz S, Malik SA, Ahmed NR, Malik SA, Farooq $S$ et al. Frequency of Congenital Hearing Loss In Neonates. J Ayub Med Coll Abbottabad. 2018;30(2):234-236.

9. Qadeer S, Junaid M, Sobani ZA, Nadeem N, Awan MS. Cochlear implant in a patient with Mondini's deformity of the cochlea: pilot patient in Pakistan. J Pak Med Assoc. 2013;63(7):913-915.

10. Ullah S, Aslamkhan M, Rasheed A. Molecular Distribution of Deafness Loci in Various Ethnic Groups of the Punjab, Pakistan. J Coll Physicians Surg Pak. 2015;25(8):573-578. doi: 08.2015/JCPSP.573578.

11. Zanon A, Sorrentino F, Franz L, Brotto D. Gender-related hearing, balance and speech disorders: A review, Hearing, Balance and Communication. 2019;17(3):203-212. 
12. Shibazaki-Yorozuya R, Nagata S. Preferential Associated Malformation in Patients with Anotia and Microtia J Craniofac Surg. 2019;30(1):66-70. doi: 10.1097/ SCS.0000000000004915

13. Masuda S, Usui S, Matsunaga T. High prevalence of inner-ear and/or internal auditory canal malformations in children with unilateral sensorineural hearing loss. Int J Pediatr Otorhinolaryngol. 2013;77(2):228-232. doi: 10.1016/j. ijporl.2012.11.001

14. Aldhafeeri AM, Alsanosi AA. Prevalence of inner ear anomalies among cochlear implant candidates. Saudi Med J. 2016;37(10):1096-1100. doi: 10.15537/smj.2016.10.15025

15. Masuda S, Usui S. Comparison of the prevalence and features of inner ear malformations in congenital unilateral and bilateral hearing loss Int J Pediatr Otorhinolaryngol. 2019;125:92-97. doi: 10.1016/j.ijporl.2019.06.028

16. Sun B, Dai P, Zhou C. Study on 2,747 cases of inner ear malformation for its classification in patient with sensorineural hearing loss. Lin Chung Er Bi Yan Hou Tou Jing Wai Ke Za Zhi. 2015;29(1):45-47.

17. Agarwal SK, Singh S, Ghuman SS, Sharma S, Lahiri AK. Radiological Assessment of the Indian Children with Congenital Sensorineural Hearing Loss. Int. J. Otolaryngol. 2014:808759. doi: $10.1155 / 2014 / 808759$

18. El Shiekh E, Abdel-Maksoud G, Wahba H, Saber I. Assessment of imaging in congenital inner ear anomalies and its relation to cochlear implantation. Zagazig Uni Med J. 2018;24(1):9-18. doi: 10.21608/zumj.2018.12987
19. Dhanasingh A. Variations in the Size and Shape of Human Cochlear Malformation Types. Anat Rec (Hoboken). 2019;302(10):1792-1799. doi: 10.1002/ar.24136

20. Yi JS, Lim HW, Kang BC, Park SY, Park HJ, Lee KS. Proportion of bony cochlear nerve canal anomalies in unilateral sensorineural hearing loss in children. Int J Pediatr Otorhinolaryngol. 2013;77(4):530-533. doi: 10.1016/j. ijporl.2012.12.031

21. Shama SAM. Revisit to congenital anomalies of the inner ear: The spectrum of aplastic/dysplastic labyrinthine malformations (ADLM). A new concept for classification. Egypt j radiol nucl med. 2012;43(4):535-542.

\section{Authors Contribution:}

JA: Did the data collection, analysis and interpretation of results.

GS: Was responsible for writing of manuscript and critical revision and was responsible for integrity of research.

IJK: Conceived the idea and critical revision of the manuscript.

MK: Did the literature review.
Authors:

1. Dr. Jawwad Ahmed, FCPS (Otolaryngology), Associate Surgeon, Department of Otolaryngology \& Cochlear Implantation, Capital Hospital PGMI, Islamabad, Pakistan.

2. Dr. Ghulam Saqulain, FCPS (Otorhinolaryngology), Head of Department of Otorhinolaryngology \&

Cochlear Implantation,

Capital Hospital PGMI, CDA, Islamabad, Pakistan.

3. Dr. Muhammad lqbal Javed Khan, FRCS Consultant Otologist \& Skull Base Surgeon, Department of Otorhinolaryngology, Bradford Teaching Hospitals NHS Foundation Trust, England.

4. Dr. Mobeen Kausar, MPH. Deputy Medical Superintendent, Healthcare Commission Coordinator, DHQ Hospital,

Rawalpindi, Pakistan. 